\title{
Onset of Normal Field Instability in a Ferrofluid in Microgravity
}

\author{
Amelia Gear, Tessa Rundle, Justin Barhite, and Jordan Rice* \\ Department of Physics, Carthage College, Kenosha Wisconsin
}

\begin{abstract}
In the presence of a uniform, perpendicular magnetic field, a phenomenon known as normal field instability (NFI) occurs in a ferrofluid. The theory describing the NFI identifies a critical magnetic field below which no magnetically driven surface deformations occur. This critical field depends on the gravitational acceleration and, according to the theory, should disappear as local gravitational acceleration approaches zero. Previous studies have been inconclusive on the existence of a critical magnetic field in reduced gravity. For our work, we designed a payload for a suborbital rocket mission which launched through the RockSat-C program. Our experiment incorporated a ferrofluid sample and a uniform magnetic field which could be varied across a discrete range of value. During the microgravity portion of the rocket's flight, we obtained video of the ferrofluid's behavior to compare it to data taken in Earth's gravity.
\end{abstract}

\section{Introduction to Ferrofluids}

A ferrofluid is a magnetic liquid composed of nanoscale ferrous particles suspended in a low-viscosity carrier fluid. When subjected to a magnetic field, surface deformation of the ferrofluid occurs along the magnetic field lines. Normal Field Instability (NFI) is the onset of this deoformation, and occurs only when the applied magnetic field exceeds a critical value [Rosensweig, 1985]. A ferrofluid experiencing NFI can be seen in Figure 1. A typical ferrofluid will be approximately $85 \%$ carrier fluid by volume [Rosensweig, 1985]. Most ferrofluids are water or oil based. The carrier fluid must be low-viscosity so that it remains flowable, even in the presence of an applied magnetic field. A ferrofluid must also have a high magnetic susceptibility, causing a large response in reaction to an applied magnetic field [Rosensweig, 1982].

The suspended magnetic particles generally have a article density of $10^{23}$ particles per meter, and are 3-5 nm in size [Rosensweig, 1985]. The particles remain suspended in the carrier fluid due to thermal agitation, which prevents them from settling out of the fluid even in the presence of an applied magnetic field. Each particle is coated in a dispersant, which prevents the particles from sticking together, becoming large enough that they would settle out of the fluid due to magnetic and gravitational forces [Rosensweig, 1985]. The dispersant is formed out of long chain molecules, with the polar head of each molecule absorbed into the surface of the ferrous particle [Rosensweig, 1985]. The tails of the molecules, which are 10 to 20 Angstroms then surround the particle and act as an elastic cushion [Rosensweig, 1982]. These

*This project was funded by the Wisconsin Space Grant Consortium and Carthage College, and made possible through the RockSat-C program provided by the Colorado Space Grant Consortium. 


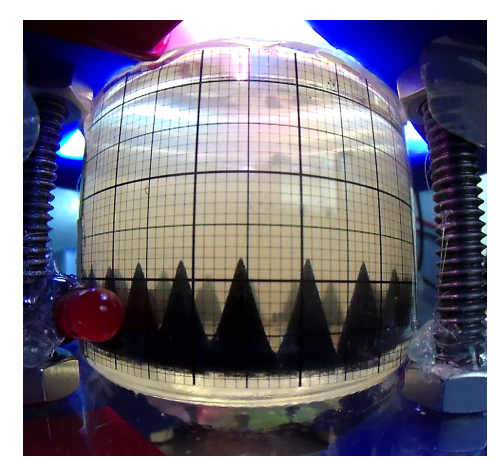

Figure 1: A ferrofluid is subjected to an external magnetic field, deforming into distinct peak and valley pattern. Due to the internal shape of the container, the fluid pools around the edges of the container.

tails are in constant motion, wiggling as the particle moves through the fluid. This motion causes the coated particles to repel one another should they come in contact [Rosensweig, 1985].

As the magnetic field increases, the magnetic dipole moments of the particles will tend to become aligned. In the presence of a strong applied magnetic field all the magnetic particles in a ferrofluid will align with the magnetic field, similar to a compass needle in an magnetic field [Rosensweig, 1982]. The magnetic field at which the particles are completely aligned and are no longer knocked out of alignment due to thermal agitation is dubbed the saturation magnetization, and this value varies with the composition of the ferrofluid [Rosensweig, 1985]. A ferrofluid will remain flowable even under saturazation magnetization [Rosensweig, 1985]. Each ferrous particle in a ferrofluid is inherently magnetized to saturation magnetization, and it is the fluid's net magnetization that is of import [Rosensweig, 1982].

Normal field instability. A ferrofluid will deform into a series of regular peaks and valleys under a uniform magnetic field applied perpendicularly to the surface of the fluid. This behavior is referred to as normal field instability (NFI). The distinct peak-and-valley pattern can be seen in Figure 1, in which the high points are the peaks and the low points, between the peaks, are the valleys. This behavior is determined by the minimization of total energy, and only occurs when the magnetic field exceeds a critical value.

Minimization of energy. The ferrofluid's patterns of deformation are governed by the minimization of energy. Under an applied magnetic field, the dominating terms in a ferrofluid's energy are the magnetic energy, gravitational energy, and surface energy. The magnetic energy drives deformation, while the surface and gravitational energy oppose it, acting as stabilizing forces to keep the surface of the ferrofluid flat [Abou, Bérengére Wesfreid, José-Eduardo Rouz, 2000]. When the magnetic field is of sufficient strength to partially overcome the surface and gravitational energies surface perturbations occur.

An individual particle will be attracted to a high magnetic field, so that particles would tend to move together to regions of strong magnetic fields. This is prevented by thermal motion, causing the particles to remain spread throughout the fluid. The necessity of having a high ratio of thermal to magnetic energy dictates maximum particle size in 


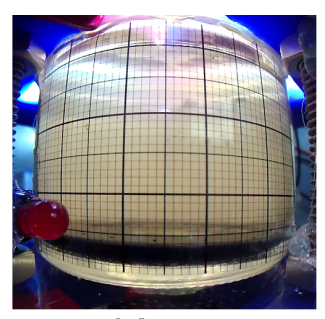

0 Gauss

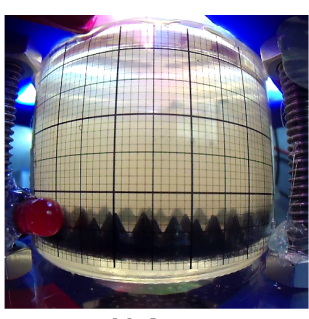

$\sim 30$ Gauss

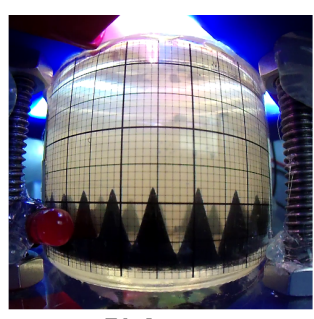

$\sim 70$ Gauss

Figure 2: A ferrofluid exposed to an increasing magnetic field. As the magnetic field increases, the peak height of the ferrofluid increases.

a ferrofluid. The small particle size prevents the particles from experiencing sufficient gravitational force to cause separation from the fluid, and each particle will experience a much stronger magnetic than gravitational force [Rosensweig, 1985].

Critical magnetic field. Deformation in a ferrofluid will only occur in the presence of a magnetic field of sufficient strength to partially overcome the stabilizing forces of gravity and surface tension. The magnetic field at which deformation occurs is referred to as the critical magnetic field. The equation for the critical magnetic field is shown in Equation 1 [Chong et al., 2012].

$$
H_{\text {crit }}=\sqrt{\frac{(1+\chi)(2+\chi) \sqrt{g \rho \sigma}}{\chi^{2} \mu_{0}}}
$$

In Equation 1, $\sigma$ denotes surface tension, $\rho$ fluid density, and $\chi$ the susceptibility of the ferrofluid. The term $g$ refers to the gravitational acceleration that the ferrofluid is subjected to, and $\mu_{0}$ delineates the permeability of free space, which has a value of $4 \pi * 10^{-7} \mathrm{Vs} / \mathrm{Am}$.

Before the critical field is reached, no surface perturbations occur. Thus, the magnetic field can be varied over a range of magnetic fields up to the critical field value, and the surface of the ferrofluid shall remain flat[Rosensweig, 1985]. The ferrofluid deforms abruptly once the magnetic field reaches this value.

Ferrofluids in varying magnetic fields. As the magnetic field increases, the wavelength of the ferrofluid, which corresponds to the distance between peaks, decreases. At the critical magnetic field the ferrofluid has a critical wavelength, which corresponds to the critical wavenumber, calculated as shown below in Equation 2.

$$
k_{c r i t}=\frac{2 \pi}{\lambda_{c}}=\sqrt{\frac{\rho g}{\sigma}}
$$

At the critical magnetic field, the surface of an infinite, thin layer of ferrofluid will deform, forming a single wave along the surface. As the magnetic field increases, more peaks will form, and under a uniform magnetic field will be uniformly spaced. An increasing field will also cause the peak height of the ferrofluid to increase. This behavior is pictured in Figure 2. 
Ferrofluids in varying gravitational fields. The ferrofluid's behavior is determined by the minimization of gravitational, surface, and magnetic energy. The gravitational and surface forces act as stabilizing forces against surface perturbations [Abou, Bérengére Wesfreid, José-Eduardo Rouz, 2000]. Surface deformation and the onset of NFI only occurs past the critical field at which the magnetic force exceeds the stabilizing forces. Gravitational forces play a key role in stabilizing the surface of the ferrofluid against surface deformation, and determining the value of the critical field. As the gravitational term in the critical magnetic field equation varies, so too does the critical magnetic field.

As the gravitational field decreases, the value of the critical magnetic field will also decrease. As the gravitational field increases, the critical magnetic field shall increase proportionally. Theoretically, the critical magnetic field should go to zero as the gravitational field approaches zero, and thus no critical magnetic field should exist in microgravity environments.

The study of the onset of NFI in ferrofluids in microgravity environments has been limited. Though studies of the behavior of a ferrofluid under the influence of a permanent magnet in microgravity and hypergravity have been carried out[Chong et al., 2012], they do not allow for observation of a ferrofluid's behavior under arbitrarily small increases of magnetic fields, under which conditions one may examine the onset of NFI. Additionally, literature is inconclusive on the existence of this critical field in microgravity. The existence of the critical magnetic field in microgravity is crucial in understanding the behavior of ferrofluids in microgravity environments and developing space-based ferrofluid applications.

Ferrofluid applications. Ferrofluids can be caused to form a variety of complex shapes due to their magnetic response[Rosensweig, 1985]. NFI causes the formation of peaks, but labyrinth patterns may also be imposed in thin layers of fluid [Chong et al., 2012]. On earth, their complexity allows ferrofluids to be used in a variety of commercial, scientific, and medical applications [Rosensweig, 1985], which could be applied to microgravity technology. In microgravity conditions, applications dealing with propulsion of small-scale satellites are under development. The peaks that ferrofluids are capable of assuming allow the substance to emit jets, which are capable of propelling small objects. They may also be used in the development of magnetic field based micro/nanoelectromechanical systems, including nanopumps, nanogenerators, and nanoactuators[Zahn, 2001].

\section{Methods}

The RockSat program. In order to observe the behavior of a ferrofluid and test the existence of a critical magnetic field in microgravity conditions, an experiment must be developed to subject the ferrofluid to microgravity conditions. This requirement is fulfilled by development of a sounding rocket payload as part of the 2014 and 2015 RockSat program. The program allows student led teams to develop a sounding rocket payload for launch aboard a Terrior-Orion rocket, subjecting the payloads to approximately 5 minutes of microgravity time.

Payload design. Our payload had three main tasks: subject a ferrofluid sample to a time-varying uniform magnetic field, record video of the ferrofluid, and measure the magnetic field produced. To accomplish this, we placed a capsule containing the 


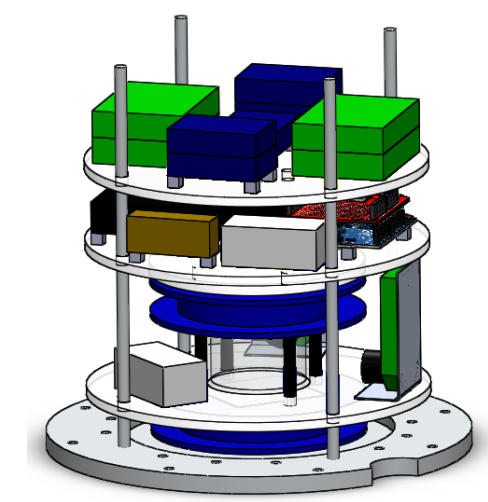

Figure 3: The internal structure of the sounding rocket payload. The Helmholtz coils are in blue, and bracket the clear ferrofluid capsule. A pair of cameras mounted to steel brackets record video of the ferrofluid's activity.

ferrofluid between a pair of Helmholtz coils, which produce a fairly uniform magnetic field between them which is proportional to the current driven through them.

The payload must comply with the Users Guide Compliance mandates as set by the RockSat program. The inner structure, which is that contained within a secondary containment structure, is composed of the electronics, Helmholtz coils, and ferrofluid capsule. All elements are mounted to polycarbonate base plates, which are light yet able to withstand the forces they are subjected to. These base plates are mounted to a system of threaded steel rods, which screw into the secondary base plate and protrude through the top of secondary containment, allowing them to be secured at both ends. The Helmholtz coils and ferrfluid capsule arrangement are mounted at the base of secondary, with two electronics plates mounted immediately above. An image of this design is found in Figure 3.

In order for accurate data on peak height to be obtained, a graph is affixed to the outer surface of the ferrofluid capsule. The graph is printed on transparent plastic so that it does not interfere with visibility of the peaks. The graph is marked in 1-millimeter intervals, with markers also clearly delineating every 5 millimeters and every centimeter. The graph over the container allows accurate measurements to be determined- correcting for fish-eye lens and curvature of the capsule.

Electronics Design. The payload electronics are controlled by an Arduino Uno, which both signals the various processes and records information from the various sensors. An accelerometer is used to record the spin and acceleration of the rocket during flight so that the behavior of the ferrofluid can be understood. A pair of magnetometers are used to record the magnetic field in the region of the ferrofluid capsule.

The Arduino Uno also controls the applied magnetic field of the payload, which is supplied by a switched-mode power supply. The switched-mode power supply outputs a signal at varying frequencies, which creates a current of varying strength. This allows the magnetic field to be continuously varied over a range, spanning 0 to 50 Gauss.

In order to have a second method of tracking the magnetic field, we added a simple 
voltage divider between the Helmholtz coil battery pack and ground, allowing the Arduino to read the battery voltage (or rather, a fixed fraction of the battery voltage) through one of its analog in pins. We also placed a thermistor in contact with one of the Helmholtz coils and set up another voltage divider so that the Arduino could read its resistance through another analog in pin. The thermistor allows us to determine estimate any change in the resistance of the Helmholtz coils due to temperature variations.

Software Subsystem. Upon activation, the Arduino waits until the second-stage burns out, then begins activating the other components; it initializes the magnetometer and turns on the cameras (by flipping the relay that connects the camera button pins to ground). After waiting a few seconds for the cameras to begin recording, the Arduino turns the MOSFET on for five seconds, applying the full voltage of the battery pack to the Helmholtz coils to produce the strongest possible magnetic field (around 70 gauss). This is done in an attempt to condense the ferrofluid in case it has been dispersed by the vibration during launch. After the five seconds, the field is turned off, and then gradually ramped up by incrementing the PWM signal from 0 to 255 over the course of five seconds. During the ramp, the Arduino periodically reads from the magnetometer, as well as the battery voltage and thermistor analog pins. Once the ramp is completed, the field is turned off, and all of the readings taken during the ramp are written to the SD card. The coil voltage is then ramped up similarly over 10 seconds, then a third time over 30 seconds. At this point the cameras are signaled to stop recording and are powered off. This entire procedure (turn cameras on, ramp up over 5, 10, and 30 seconds, and turn cameras off) is repeated two more times, for a total of three cycles.

Ferrofluid. For the purposes of this experiment, approximately $1 / 4$ of an ounce of light hydrocarbon ferrofluid was used. The remaining space in the two-ounce ferrofluid capsule is occupied by a suspension fluid, composed mainly of water. The suspension fluid prevents the ferrofluid from staining the glass capsule. Both the suspension fluid and ferrofluid were provided by ConceptZero. The ferrofluid used is Ferrotec's EFH1, developed for educational markets with well-documented physical properties.

Testing results. Throughout the initial design and construction process last year, we tested all of our components to verify their correct functioning and to determine optimal placement within the payload. For instance, we ran a large current through our Helmholtz coils for an extended period of time to verify that they wouldn't dissipate enough heat to cause any issues. We tested various placements of the cameras and adjusted their focus to get the best view of the ferrofluid sample and the grid lines on the outside of the container. We also tested various placements of the LEDs to find the optimal position for lighting the ferrofluid while avoiding glare on the glass container. The magnetometer placement had to be investigated as well, since its range of $+/-8$ gauss meant that it would saturate if placed too close to the center of the coils. The magnetometer was also tested by attaching it to one of the electronics plates above the Helmholtz coils, and calibrations were done against the magnetic field at the center of the coils at the location of the ferrofluid capsule.

Finally, we ran numerous full mission simulations to verify that all components were properly integrated and that the payload performed as expected. The first full mission simulations were conducted on March 26th, and more were run on several occasions 

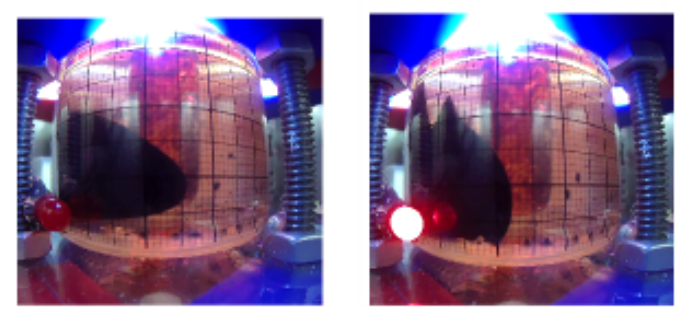

Figure 4: A comparison of the ferrofluid bubble in microgravity with no applied magnetic field (left) and with an applied field of 50 Gauss (right).

between then and launch. Most full mission simulations were run without secondary containment, so that the g-switch could be manually triggered and the payload could be observed as it ran. In addition to verifying proper integration of all parts, we also used the full mission simulations to test possible failure scenarios, such as removal of the SD card or reset of the Arduino. These tests demonstrated that the payload was able to recover in both of those cases. We also ran a few full mission simulations with the payload spinning at approximately $2 \mathrm{~Hz}$ in order to see what effect the spin would have on the payload, and on the ferrofluid in particular. While this was significantly less than the spin of the rocket during flight, we saw no difference in the ferrofluid's behavior spinning at $2 \mathrm{~Hz}$.

Observing the onset of normal field instability. In order to compare the onset of NFI in microgravity to that in earth-gravity conditions, ground data must be obtained. To do this, video of the ferrofluid subjected to a range of magnetic fields is obtained on Earth. Video was recorded, allowing the surface of the ferrofluid to be observed for deformation.

The onset of NFI is determined to occur when deformation is first seen. To do this, the surface of the ferrofluid was visually analyzed at each magnetic field level. At the point when a peak first begins to form, the applied magnetic field is obtained from the magnetometer data, and the height of the ferrofluid surface was recorded.

\section{Results}

Our payload operated as expected during flight. Both cameras recorded the expected three videos, and the observed deformations of the ferrofluid indicate that the Helmholtz coils were supplied with the intended currents. The Arduino recorded reasonable values for the battery voltage, thermistor resistance, and magnetometer, suggesting that those components operated correctly, and the timestamps logged to the SD card show that the Arduino did not reset during the portion of the flight when the experiment was active.

The images in Figure 4 are taken from the second video recorded by one of the cameras during flight. In the first image no magnetic field is present, and in the second a field of about 50 gauss is applied to the ferrofluid. At the bottom left one of the signal LEDs is visible, which allows frames in the videos to be correlated with timestamps recorded by the Arduino. The air bubble in the container is visible at the center (due to the spin of the rocket). 


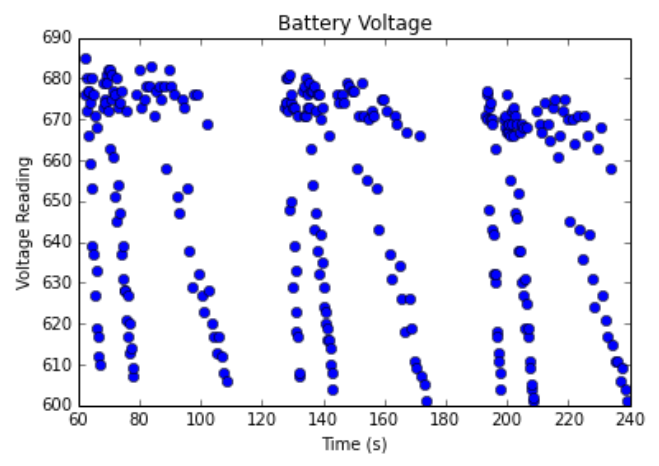

Figure 5: A plot of the battery pack voltage during flight. This was the primary source of data for calculating the magnetic field experienced by the ferrofluid.

Figure 5 shows the voltage of the battery pack supplying current to the Helmholtz coils throughout the duration of the experiment. The voltage data are analog readings from the Arduino, which we calibrated against the actual battery voltage prior to flight. During each of the three cycles, the battery voltage drops three times, over 5, 10, and 30 seconds. This occurs because the current to the coils is being ramped up; consequently the battery voltage drops due to the battery's internal resistance. The average battery voltage also decreases slightly over time as it is gradually discharged by powering the coils. Overall, this plot gives us confidence that the Helmholtz coils were supplied with the correct currents and that there were no power failures.

We also recorded readings from the thermistor placed in contact with one of the coils; the data shows a steady decrease in the thermistor's resistance throughout the experiment, corresponding to an increase in temperature resulting from the heat dissipated by the coils as a current is driven through them. The calibration we performed before flight indicates that the observed change in the thermistor readings does not correspond to a large enough temperature change to significantly affect the coils' resistance. Therefore we can conclude that any changes in the coils' resistance due to temperature variations can be ignored when determining the magnetic field present throughout the flight.

The magnetometer data, unfortunately, is not as useful. In Figure 6, the red, blue, and green points show the measurements of the magnetic field in the $\mathrm{x}, \mathrm{y}$, and $\mathrm{z}$ directions (which are determined by the orientation of the magnetometer). Two of these channels were vertically offset in the plot so they would not overlap. The solid line is proportional to the PWM signal to the MOSFET, which in turn is approximately proportional to the magnetic field. The green and blue channels appear to decrease linearly with increasing magnetic field at low fields, but the blue channel quickly saturates and the green channel starts trending upwards. The red channel also does not have any strong correlation with the magnetic field.

Since the ferrofluid did not form a flat layer during the flight, we were not able to test the theoretical predictions of the critical field and wave number of the Rosensweig instability. However, some of the videos showed small, nearly spherical droplets of ferrofluid which were elongated as the field increased. The images in Figure 7 show one of these droplets at different applied fields. 


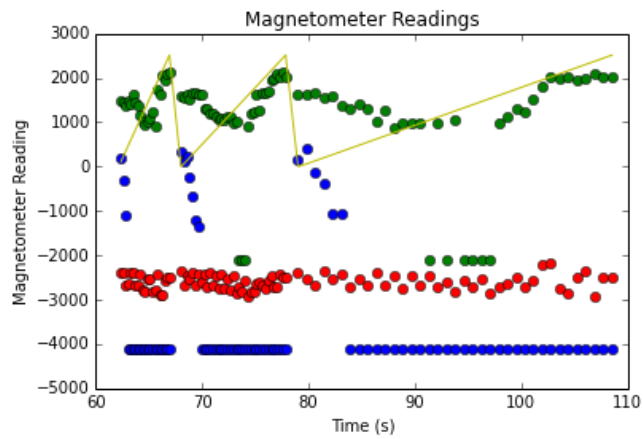

Figure 6: The data recorded by the magnetometer during flight.

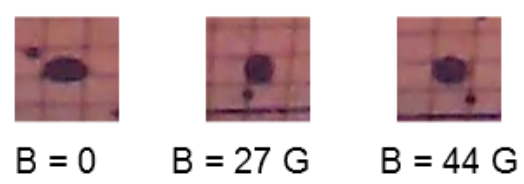

Figure 7: A ferrofluid droplet experiencing various magnetic fields in microgravity.

We modeled these droplets theoretically in order to compare our predictions to their observed deformations under the applied field. We did so by considering the magnetic energy and surface energy of a prolate ellipsoid of a uniformly magnetized material with a given volume, surface tension, and magnetic susceptibility. We were able to show that for small deviations from a sphere, as the eccentricity of the ellipsoid increases (at constant volume), the magnetic energy decreases as the square of the eccentricity while the surface energy increases as the fourth power of the eccentricity. Thus the sphere is unstable under any nonzero applied field; while an ellipsoid may not be the true shape of the deformed droplet, since it is energetically preferable to the sphere, the droplet must deform into some other shape. Furthermore, by finding the eccentricity that minimizes the total energy, we can predict the approximate shape that a small droplet would adopt under a given applied field.

For the droplet shown in the images above, we estimated the ratio of the axes of the ellipsoid every five frames while the field was increasing, and plotted the axis ratios against the applied field. On this plot, Figure 8 we then superimposed the theoretical prediction of the axis ratio as a function of applied field, using values of the susceptibility and surface tension specific to our ferrofluid.

The discrepancy between the theoretical predictions and the observed behavior of the ferrofluid droplet in the video could result from distortion of the droplet by the camera, the centrifugal force pressing the droplet against the glass, a non-ellipsoidal true shape of the droplet, or a slight inaccuracy in our estimation of the magnetic field. Considering the number of possible sources of error, the plot shows fairly good agreement between data and theory.

\section{Conclusions}

Our payload performed as intended during flight and collected the expected amount 


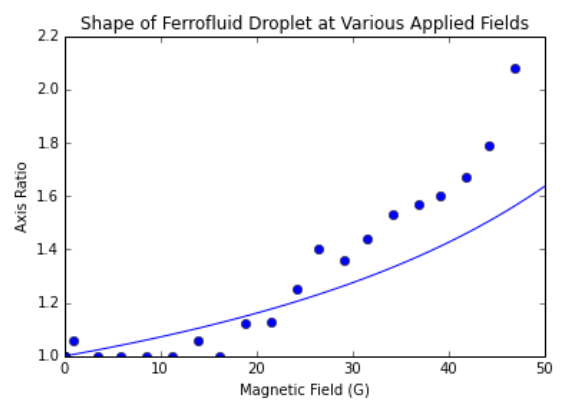

Figure 8: The theoretical prediction of the axis ratio of a drop of ferrofluid at various applied magnetic fields, superimposed with the measurements of an experimental droplet.

of data, so the operation of our payload was a success. Since the ferrofluid did not form a flat layer perpendicular to the magnetic field during flight, we did not observe the Rosensweig instability in the videos and were therefore unable to characterize the role of gravity in stabilizing the surface of the fluid against magnetically driven deformations. However, due to the presence of several small, isolated, nearly spherical droplets of ferrofluid, we were able to validate our theoretical modeling of the effects of magnetic and surface energy in determining the shape of a small droplet of ferrofluid in the absence of gravity.

Potential Follow-up Work Several improvements could be made if this experiment were to fly again. Other magnetometers and different placements of the magnetometer could be explored to obtain more reliable measurements of the magnetic field. As another method of tracking the magnetic field, a simple ammeter could be implemented; measurements of the current in the coils would likely correlate better with the field than battery voltage multiplied by the PWM duty cycle.

\section{References}

[Abou, Bérengére Wesfreid, José-Eduardo Rouz, 2000] Abou, Bérengére Wesfreid, José-Eduardo Rouz, S. (2000). The normal field instability in ferrofluids: hexagon-square transition mechanism and wavenumber selection. Journal of Fluid Mechanics, 416:217-237.

[Chong et al., 2012] Chong, T. Y., Ho, K. L., and Ong, B. H. (2012). Investigations of field instability of ferrofluid in hypergravity and microgravity. AIP Advances, 2(1):012138.

[Rosensweig, 1982] Rosensweig, R. (1982). Magnetic Fluids. Scientific American, 247(4):136-145.

[Rosensweig, 1985] Rosensweig, R. E. (1985). Ferrohydrodynamics. Cambridge University Press.

[Zahn, 2001] Zahn, M. (2001). Magnetic fluid and nanoparticle applications to nanotechnology. pages $73-78$. 